\title{
Neutron capture studies with a short flight path
}

\author{
Stephan Walter ${ }^{* 1}$, Michael Heil ${ }^{2}$, Franz Käppeler ${ }^{1}$, Ralf Plag $^{1}$, and René Reifarth \\ ${ }^{1}$ Institut für Kernphysik, Forschungszentrum Karlsruhe, Postfach 3640, D-76021 Karlsruhe, \\ Germany \\ ${ }^{2}$ Gesellschaft für Schwerionenforschung, Planckstr. 1, D-64291 Darmstadt, Germany \\ ${ }^{3}$ Los Alamos National Laboratory, Los Alamos, New Mexico, 87545, USA \\ E-mail: Stephan.walter@ik.fzk.de
}

\begin{abstract}
The time of flight (TOF) method is an important tool for the experimental determination of neutron capture cross sections which are needed for s-process nucleosynthesis in general, and for analyses of branchings in the s-process reaction path in particular. So far, sample masses of at least several milligrams are required to compensate limitations in the currently available neutron fluxes. This constraint leads to unacceptable backgrounds for most of the relevant unstable branch point nuclei, due to the decay activity of the sample. A possible solution has been proposed by the NCAP project at the University of Frankfurt. A first step in this direction is reported here, which aims at enhancing the sensitivity of the Karlsruhe TOF array by reducing the neutron flight path to only a few centimeters. Though sample masses in the microgram regime can be used by this approach, the increase in neutron flux has to be paid by a higher background from the prompt flash related to neutron production. Test measurements with Au samples are reported.
\end{abstract}

International Symposium on Nuclear Astrophysics - Nuclei in the Cosmos - IX

25-30 June 2006

CERN

${ }^{*}$ Speaker. 


\section{The s process and neutron capture cross sections}

Neutron capture reactions in the s process contribute to about $50 \%$ of the isotopic abundances between Fe and Bi. Since the time span between two neutron capture reactions (1-10 years) is much longer than the time scale for $\beta^{-}$decays, the reaction path follows closely the valley of stability.

Sometimes radioactive isotopes with half-lives comparable to the time between two neutron capture reactions are encountered, leading to a branching in the nucleosynthesis path. The knowledge of the corresponding $(\mathrm{n}, \gamma)$-cross sections allows one to obtain information about the physical conditions in the s process during stellar helium burning [1], 2], but are also important for the nucleosynthesis in the $r$ and $p$ process during supernova explosions [3].

The neutron capture cross sections can be measured via the activation method [ 4 ] or the time of flight (TOF) technique. In activation experiments, one obtains the Maxwellian averaged cross section (MAC), whereas in TOF measurements the energy dependent cross section can be extracted over a certain energy range.

\section{The Karlsruhe $4 \pi \mathrm{BaF}_{2}$-detector}

In TOF experiments, total absorption calorimeters (TAC) are meanwhile used in different places, taking advantage of the high efficiency and good timing properties of the detector crystals. The Karlsruhe $4 \pi$ calorimeter consists of $42 \mathrm{BaF}_{2}$ crystals, and the nominal flight path between the neutron production target and the sample is $77 \mathrm{~cm}$. Neutrons are produced via the ${ }^{7} \mathrm{Li}(\mathrm{p}, \mathrm{n})^{7} \mathrm{Be}$ reaction, using a pulsed proton beam with an energy of about $2 \mathrm{MeV}$. For background reduction, the target is surrounded by a lead enforced collimator, which reduces the neutron beam to a diameter of about $20 \mathrm{~mm}$ [5].

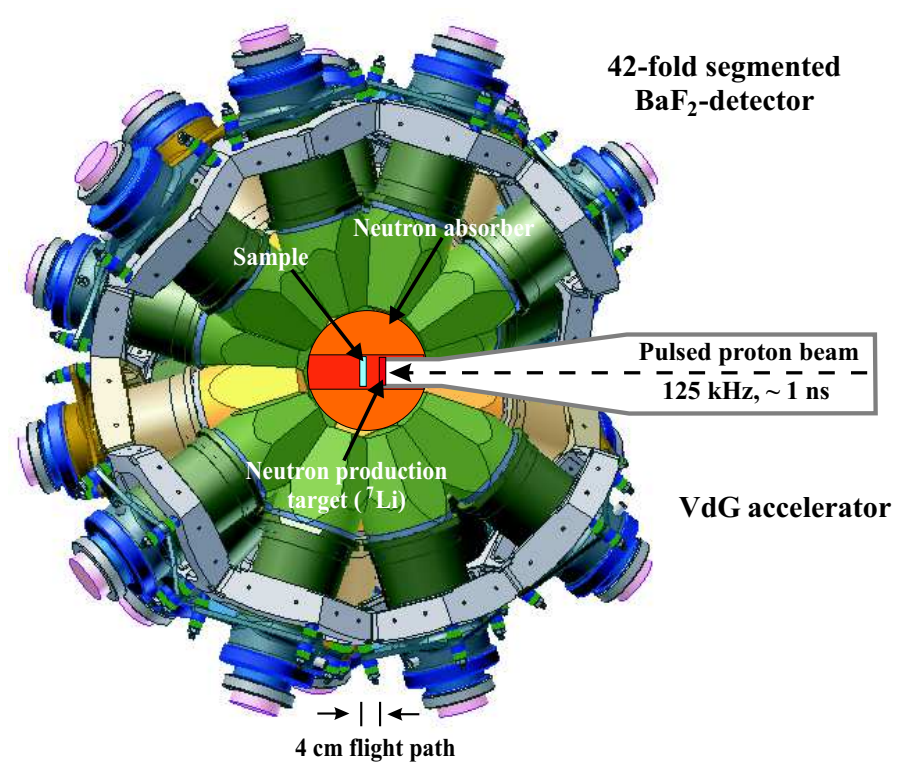

Figure 1: New setup of the Karlsruhe $4 \pi$-detector: the distance between the ${ }^{7} \mathrm{Li}$ neutron production target and the sample is reduced to $4 \mathrm{~cm}$, leading to an expected increase in neutron flux by two orders of magnitude. 
Radioactive samples can only be used in very small quantities - otherwise the background due to the activity of the target would be too high. For the measurement of such samples, the sensitivity of the setup needs to be increased.

One possibility is to move the neutron production target inside the $4 \pi$ detector as shown in Fig. 1. The reduction in flight path from $77 \mathrm{~cm}$ down to $4 \mathrm{~cm}$ leads to an expected increase in neutron flux by two orders of magnitude. This would allow to use sample masses in the microgram region.

The drawback of this modification is a higher background - mainly due to the $\gamma$-flash when the proton beam hits the neutron production target. In an idealized picture, a time window between the prompt $\gamma$-rays and the background from scattered and captured neutrons inside the detector material should allow undisturbed measurements. For $100 \mathrm{keV}$ neutrons, this time window would start $9 \mathrm{~ns}$ after the $\gamma$-flash, ending $17 \mathrm{~ns}$ later when the fastest neutrons reach the detector material.

\section{Background reduction and first test measurements}

The background has two different origins: the $\gamma$-flash itself and $\gamma$-rays produced by scattered or captured neutrons inside the detector material.

The amount of photons that are produced in the $\gamma$-flash depends on the backing material of the Li target, and on the thickness of the lithium layer itself. While $\mathrm{Cu}$ was used in the past as backing due to its high heat conductivity, Ta will be used for the short flight path, resulting in a reduction in the number of produced photons by a factor of about 400 .

However, by far most of the $\gamma$-rays are produced in the lithium layer (about a factor 150 more than in $\mathrm{Cu}$ ). Since ${ }^{7} \mathrm{Li}$ is needed for neutron production, the only way of reducing the background is a reduction in $\mathrm{Li}$ layer thickness. For $5 \mu \mathrm{m}$ of $\mathrm{Li}$, the protons are still slowed down from $30 \mathrm{keV}$ above the neutron production threshold at $1.881 \mathrm{MeV}$ below the threshold, thus resulting in a quasi Maxwellian neutron energy distribution of $25 \mathrm{keV}$. The expected count rate using such a Li layer the accelerator working with a pulse repetition rate of $125 \mathrm{kHz}$ and $1.5 \mu \mathrm{A}$ beam current - is 1-2 $\gamma$-rays per pulse and per crystal.

Several possibilities for further background reduction were considered as well. The scintillation light of $\mathrm{BaF}_{2}$ crystals consists of two components: one with a peak emission at $\lambda=220 \mathrm{~nm}$ and a decay time constant $\tau=0.6 \mathrm{~ns}$, the other with $\lambda=310 \mathrm{~nm}$ and $\tau=620 \mathrm{~ns}$ [6]. For high count rate applications, only the fast component is of interest. The fast to slow ratio can be influenced by the crystal temperature. Increasing the temperature from $20^{\circ} \mathrm{C}$ to $65^{\circ} \mathrm{C}$ increases this ratio by about a factor of 1.6, but such high crystal temperatures are not suited for the real measurements.

Additionally, the possibility of using UV-filters or wavelength shifters such as p-Terphenyl was also considered. It turned out that these devices would either reduce the signal strength too much or would influence both components - more or less - equally. Therefore, these ideas had to be abandoned.

Although passive shieldings inside the detector are difficult for geometrical reasons, the use of a lead absorber around the Li target was investigated and the influence on the $\gamma$-rays both from the target and the sample was simulated with the GEANT4 toolkit [7].

The lead shield consists of a massive sphere $66 \mathrm{~mm}$ in diameter with two openings: a cylindrical on one side for the beamline, and a cone with $120^{\circ}$ opening angle on the other side in order to 

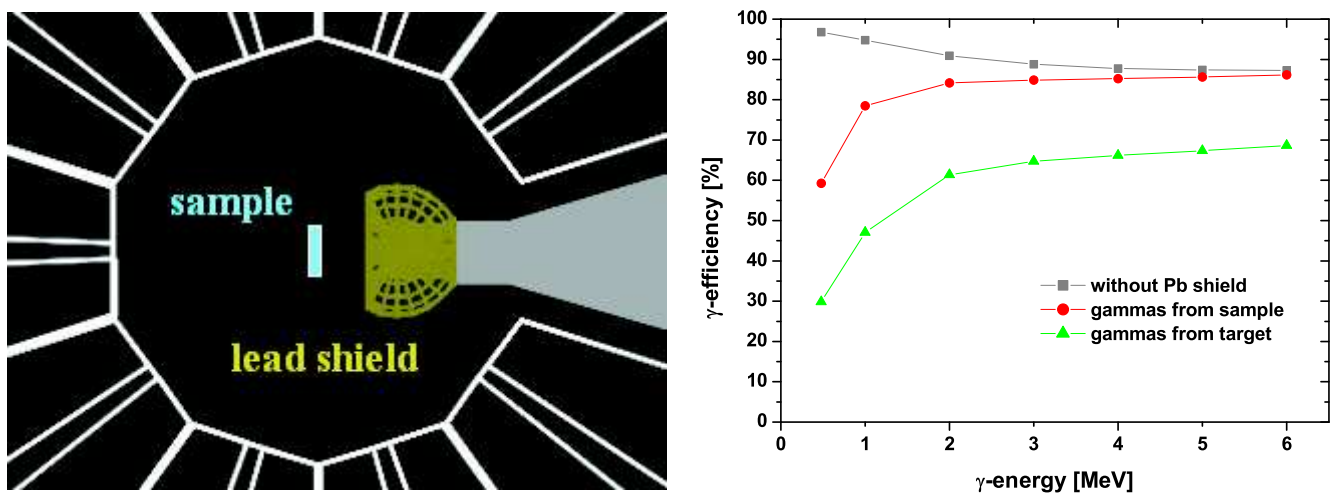

Figure 2: In order to reduce the background, the ${ }^{7} \mathrm{Li}$ target can be surrounded by a lead shield (with an opening for the beamline and the neutrons opposite to each other). The influence on $\gamma$-rays with different energies was simulated with GEANT4: the upper curve shows the results without the Pb shield (squares), the curve in the middle the influence on $\gamma$ 's from the sample (circles) and the lower curve on $\gamma$ 's from the target (triangles).

avoid interactions of the neutron beam with the shielding (Fig. 2). For $\gamma$-ray energies of $2 \mathrm{MeV}-\mathrm{a}$ typical capture $\gamma$-ray energy considering a Q-value of $6 \mathrm{MeV}$ and a multiplicity of 3 - the number of photons from the target are reduced by $30 \%$, whereas sample $\gamma$-rays are only reduced by $7 \%$.

In order to verify the simulated properties of the new setup, test measurements with a gold sample have been performed. Time of flight spectra showed that the $\gamma$-flash is clearly separated from the rest of the spectrum. Further test measurements concerning the influence of a neutron absorber inside the $4 \pi$-detector in addition to the lead shield, but also background subtraction and data reduction are planned for the near future.

\section{4. ${ }^{63} \mathrm{Ni}$}

The s process consists of two components: the main component, responsible for isotopes with A $>90$ that occurs in the He-rich intershell of thermally pulsing low mass asymptotic giant stars, and the weak component, that contributes to the isotopes with $56<\mathrm{A}<90$, and operates during the convective core He burning in massive stars. Especially neutron capture cross sections in the latter mass region have to be very accurate since the local approximation $\langle\sigma\rangle N=$ const is not valid any more. Changes in the capture cross sections can therefore have a large impact on the abundances of the heavier isotopes [8].

In the case of the Ni isotopes, it was not clear if the resulting overabundances in stellar model calculations are due to residual uncertainties in these models or to inaccuracies of the nuclear physics input [9]. Recent activation measurements showed the influence of different $(n, \gamma)$-cross sections on the nucleosynthesis yield in the case of ${ }^{62} \mathrm{Ni}$ [10], but also TOF measurements on the same isotope have been performed [11, 12].

Figure 3 shows a part of the chart of nuclides around ${ }^{63} \mathrm{Ni}$. This isotope is the first branching point in the weak s-process path. Hence, its decay and neutron capture rates are directly influencencing the abundance of ${ }^{63} \mathrm{Cu}$. Up to now, only theoretical predictions with fairly large uncertainties exist for this $(\mathrm{n}, \gamma)$-cross section $(\operatorname{MACS}(30)=31(6) \mathrm{mb}[13])$. 


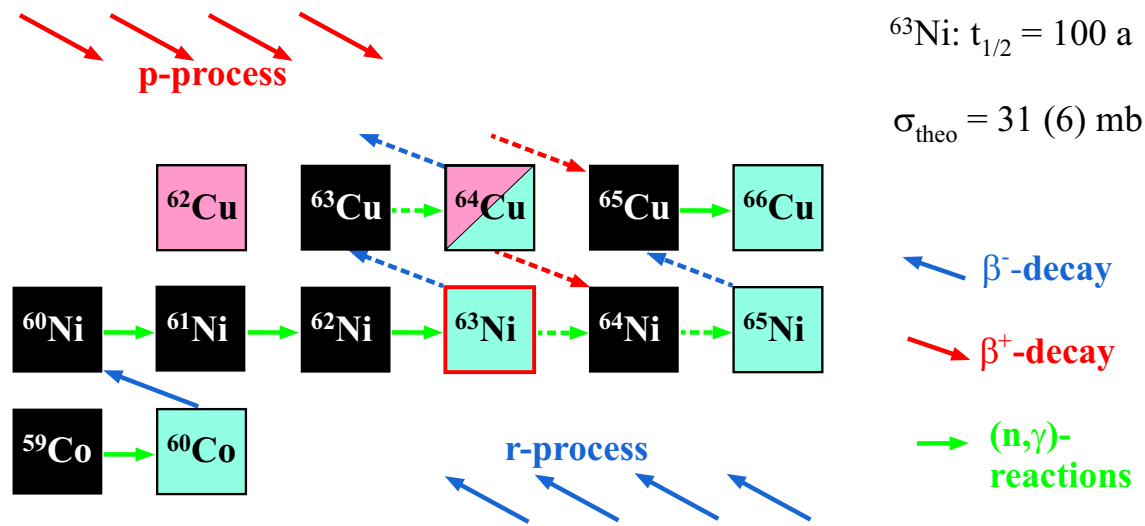

Figure 3: ${ }^{63} \mathrm{Ni}$ is the first branching point in the s process path. The branching has direct influence on the abundance of ${ }^{63} \mathrm{Cu}$.

First TOF measurements with the new setup of the Karlsruhe $4 \pi$-detector are planned as soon as the necessary test measurements and the verification phase have been completed.

\section{References}

[1] F. Käppeler, Nuclear Physics A 752, 500c-509c (2005).

[2] F. Käppeler, M. Wiescher, U. Giesen, J. Görres, I. Baraffe, M. E. Eid, C. M. Raiteri, M. Busso, R. Gallino, M. Limongi, and A. Chieffi, Ap. J. 437, 396-409 (1994).

[3] S. E. Woosley, A. Heger, and T. A. Weaver, Rev. Mod. Phys. 74, 1015-1071 (2002).

[4] W. Ratynski and F. Käppeler, Phys. Rev. C 37, 595-605 (1988).

[5] K. Wisshak, K. Guber, F. Käppeler, J. Krisch, H. Müller, G. Rupp, and F. Voss, Nucl. Instr. Meth. A 595-618 (1990).

[6] M. Laval, M. Moszyński, R. Allemand, E. Cormoreche, P. Guinet, R. Odru, and J. Vacher, Nucl. Instr. Methods 206, 169-176 (1983).

[7] S. Agostinelli, J. Allison, K. Amako, J. Apostolakis, H. Araujo, P. Arce, M. Asai, D. Axen, S. Banerjee, G. Barrand, F. Behner, L. Bellagamba et. al, Nucl. Instr. Meth. A 506, 250-303 (2003).

[8] M. Heil, and M. Pignatari AIP Conf. Proc. 819, 265-272 (2006).

[9] T. Rauscher, A. Heger, R. D. Hoffman, and S. E. Woosley Astrophys. J. 576, 323-348 (2002).

[10] H. Nassar, M. Paul, I. Ahmad, D. Berkovits, M. Bettan, P. Collon, S. Dababneh, S. Ghelberg, J. P. Greene, A. Heger, M. Heil, D. J. Henderson, C. L. Jiang, F. Käppeler, H. Koivisto, S. O'Brien, R. C. Pardo, N. Patronis, T. Pennington, R. Plag, K. E. Rehm, R. Reifarth, R. Scott, S. Sinha, X. Tang, and R. Vondrasek Phys. Rev. Lett. 94, 092504 (2005).

[11] A. Tomyo, Y. Temma, M. Segawa, Y. Nagai, H. Makii, T. Shima, T. Ohsaki, and M. Igashira Astrophys. J. 623, L153-L156 (2005).

[12] A. M. Alpizar-Vicente, T. A. Bredeweg, E.-I. Esch, U. Greife, R. C. Haight, R. Hatarik, J. M. O'Donnell, R. Reifarth, R. S. Rundberg, J. L. Ullmann, D. J. Vieira, J. B. Wilhelmy, and J. M. Wouters AIP Conf. Proc. 819, 273-277 (2006).

[13] Karlsruhe Astrophysical Database of Nucleosynthesis in Stars (KADoNiS), http://nuclear-astrophysics.fzk.de/kadonis 